\title{
Plasmonic properties of superconducting niobium in the optical part of the spectrum
}

\author{
C. Y. Liao ${ }^{1,2}$, H. N. S. Krishnamoorthy ${ }^{3}$ V. Savinov ${ }^{l}$, J. Y. Ou ${ }^{l}$, C. Huang ${ }^{3}$, G. Adamo ${ }^{3}$, E. Plum ${ }^{l}$, K. F.

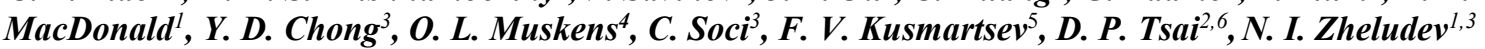 \\ 1. Optoelectronics Research Centre \& Centre for Photonic Metamaterials, University of Southampton, SO17 1BJ, UK \\ 2. Department of Physics, National Taiwan University, Taipei 10617, Taiwan \\ 3. Centre for Disruptive Photonic Technologies, TPI, SPMS, Nanyang Technological University, Singapore 637371 \\ 4. School of Physics and Astronomy, University of Southampton, SO17 1BJ, UK \\ 5. Department of Physics, Loughborough University, Loughborough, LE11 3TU, UK \\ 6. Research Center for Applied Sciences, Academia Sinica, Taipei 115, Taiwan
}

We show for the first time that, contrary to common expectations, transition to superconductivity affects plasmonic behaviour of niobium at optical frequencies. This result is unexpected as photon energy is orders of magnitude higher than the binding energy of the Cooper pairs, the superconducting charge carriers.

We study temperature-induced variation in the optical properties of a nanostructured superconducting metamaterial. In non-superconducting metamaterials, temperature-related changes in optical response tend to saturate below $50 \mathrm{~K}$. In contrast, both the position and the strength of our metamaterial's resonances exhibit a pronounced critical dependence on temperature down to a few Kelvin. Niobium is a well-known lowtemperature superconductor, well-described by the Bardeen-Cooper-Schrieffer theory. Our results shed new light on this material and theory, suggesting that superconductivity may play a role in optical dielectric response near the superconducting transition.
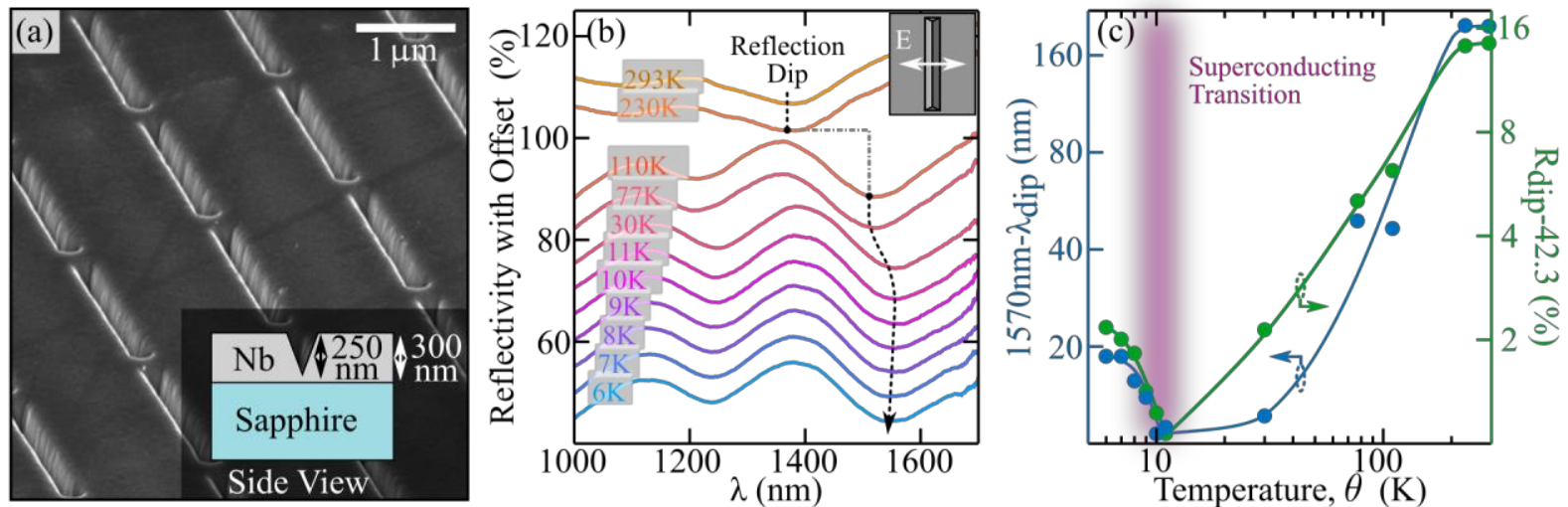

Fig. 1. Superconducting near-infrared metamaterial. (a) SEM picture of the manufactured niobium (Nb) V-groove metamaterial with composition and geometry shown inset. (b) Reflectivity spectra of the metamaterial at different substrate temperatures for light polarized perpendicular to the V-grooves (as illustrated inset; ' $E$ ' denotes electric field). Traces are sequentially offset vertically by $5 \%$ upwards for clarity. The black dashed line highlights the change in the spectral position of the long-wavelength resonant dip as a function of temperature. (c) The position (blue; left axis) and depth (green; right axis) of the long-wavelength resonant dip in metamaterial reflectivity as a function of temperature. The pink vertical stripe highlights the transition temperature of niobium.

The superconducting metamaterial (Fig. 1a), was created by focused ion beam (FIB) milling $250 \mathrm{~nm}$ deep Vgrooves in a $300 \mathrm{~nm}$ thick niobium $(\mathrm{Nb})$ film on a sapphire substrate. Niobium is a type-II superconductor with transition temperature at $9.2 \mathrm{~K}$, below which it exhibits zero DC and low microwave resistance. The binding energy of the Cooper pairs in $\mathrm{Nb}$ is just few $\mathrm{meV}$ so the response at optical frequencies will be lossy, since each optical-range photon carries sufficient energy $(\sim 1 \mathrm{eV})$ to break Cooper pairs and therefore to locally supress superconductivity.

The metamaterial was placed in an optical cryostat and its reflectivity was measured from room temperature down to $6 \mathrm{~K}$ (Fig 1b). Two broad resonant dips are observed in all spectra (Fig. 1b), one at $\sim 1100-1300 \mathrm{~nm}$ and another at 1400-1600 nm. As temperature decreases, one initially observes a red-shift in the position of the latter as well as a decrease in the depth of the reflectivity dip, however at $\sim 9-10 \mathrm{~K}$, as $\mathrm{Nb}$ enters the superconducting state, both trends are reversed. Furthermore, we also observed such a critical behaviour in the experimentally measured dielectric constants of an unstructured $\mathrm{Nb}$ film around the transition temperature. We explain the experimentally observed critical dependence of the metamaterial resonance position on the transition temperature of $\mathrm{Nb}$ by means of a thermodynamics-based model that takes into account the change in the free energy of the metamaterial resonator between the normal and superconducting states. We argue that this is a signature of the transition to the superconducting state, which is detected by infrared photons. 